\title{
Parametric amplifier for Bell measurement in continuous-variable quantum state teleportation
}

\author{
Xin Chen and Z. Y. Ou $\odot^{*}$ \\ Department of Physics, Indiana University-Purdue University Indianapolis, Indianapolis, Indiana 46202, USA
}

(Received 18 May 2020; accepted 27 August 2020; published 15 September 2020)

\begin{abstract}
A parametric amplifier is in essence a linear four-port device, which couples and linearly mixes two inputs before amplifying and sending them to two output ports. Here we show that for quadrature-phase amplitudes, a parametric amplifier can replace beam splitters to play the role of the mixer. We apply this idea to a continuousvariable quantum state teleportation scheme in which a parametric amplifier replaces a beam splitter in the Bell measurement. We show that this scheme is loss tolerant in the Bell measurement process and thus demonstrate the advantage of the parametric amplifier over the beam splitter in the applications in quantum measurement.
\end{abstract}

DOI: 10.1103/PhysRevA.102.032407

\section{INTRODUCTION}

Quantum interference plays an important role in the display of many quantum phenomena. It usually requires a linear beam splitter to superimpose two fields for interference between them. This happens in many protocols of quantum information processing. For example, optical quantum computing relies on the Hong-Ou-Mandel effect where a beam splitter is an essential element [1,2]. Current applicable schemes of Bell measurement $[3,4]$ for quantum state teleportation [5-8] require beam splitters to mix the incoming unknown state with one field of an entangled state.

It is well known that losses are notorious in degrading quantum effects and are the key obstacle in many protocols of quantum information processing. The detection process often introduces losses due to imperfect coupling and less-thanunity quantum efficiency. Highly efficient detectors are only available for some limited spectrum of the electromagnetic waves. Thus, it becomes a major concern in high-fidelity quantum communication involving quantum measurement by detection. Quantum state teleportation is one such quantum communication protocol where a Bell measurement is performed to projectively select out the required states. For continuous-variable quantum state teleportation, Bell measurement is usually achieved by homodyne detection, which is sensitive to losses. This will inevitably affect the fidelity of the teleported state.

On the other hand, amplification is known to overcome the effect of losses. Indeed, parametric amplifiers (PAs) were recently used in $\mathrm{SU}(1,1)$ interferometers [9] and quantum entanglement measurement [10] to mix two fields by replacing beam splitters (BSs) for interference and were demonstrated to be loss tolerant in detection processes.

At first look, it seems counterintuitive that a parametric amplifier can be of any use in quantum information science and play any role in mixing fields for interference since it is often portrayed as adding extra noise and thus degrading the

\footnotetext{
*zou@iupui.edu
}

signal in the amplification processes [11]. However, if we look into the origin of the extra noise, we find it comes from the vacuum fluctuations of the internal degrees of the amplifier. So if we can access these internal degrees and place them in correlation with the input, the extra noise can actually be suppressed due to quantum correlation $[12,13]$. Therefore, by treating the internal degrees of the amplifier as another input, we mix them with the original input and use the amplifier as a field mixer similar to a beam splitter. Specifically, parametric amplifiers are such devices for which the internal degree is the so-called idler field that we can easily access from outside. In essence, a parametric amplifier is a four-port linear device just like a beam splitter, even though it is often realized through nonlinear interaction with energy actively pumped into it for amplification.

In this paper we investigate the feasibility of replacing a beam splitter by a parametric amplifier for Bell measurement in a quantum teleportation scheme and demonstrate the loss tolerant property of the scheme. The paper is organized as follows. In Sec. II we introduce quantum state transformation for both the beam splitter and parametric amplifier. This is based on the Wigner representation of the quantum state. In Sec. III we present the result for quantum teleportation with a parametric amplifier and demonstrate its feasibility. The loss-tolerant property of the scheme is discussed in general in Sec. IV and its influence on the transmission of pure states such as a coherent state and photon number Fock states is discussed in Sec. V and on Einstein-Podolsky-Rosen (EPR) entangled states in Sec. VI. We conclude with a summary and discussion in Sec. VII.

\section{QUANTUM STATE TRANSFORMATION OF A PARAMETRIC AMPLIFIER}

The role played by a parametric amplifier in the mixing of fields for interference can be understood from the input-output relation

$$
\hat{a}_{1(\mathrm{PA})}^{(o)}=G \hat{a}_{1}^{(i)}+g \hat{a}_{2}^{(i) \dagger}, \quad \hat{a}_{2(\mathrm{PA})}^{(o)}=G \hat{a}_{2}^{(i)}+g \hat{a}_{1}^{(i) \dagger},
$$


where $G$ and $g$ are amplitude gains satisfying $G^{2}-g^{2}=1$ and without loss of generality we assume they are real and positive (otherwise we can always absorb the extra phases in $\hat{a}_{1,2}$ ). In comparison, the input-output relation for a beam splitter is given by

$$
\hat{a}_{1(\mathrm{BS})}^{(o)}=t \hat{a}_{1}^{(i)}+r \hat{a}_{2}^{(i)}, \quad \hat{a}_{2(B S)}^{(o)}=t \hat{a}_{2}^{(i)}-r \hat{a}_{1}^{(i)},
$$

with $t^{2}+r^{2}=1$. It can be seen that the PA is basically a fourport linear device that not only amplifies but also, similar to a beam splitter, mixes the two input fields.

However, the difference between the two devices is also obvious: The PA output is related to the Hermitian conjugate of the second input field, which can lead to unwanted spontaneous emission even with input in a vacuum, as seen in the average photon number

$$
\begin{aligned}
\left\langle\hat{N}_{1(\mathrm{PA})}^{(o)}\right\rangle & \equiv\left\langle\hat{a}_{1}^{(o) \dagger} \hat{a}_{1}^{(o)}\right\rangle \\
& =G^{2}\left\langle\hat{a}_{1}^{(i) \dagger} \hat{a}_{1}^{(i)}\right\rangle+g^{2}\left(\left\langle\hat{a}_{2}^{(i) \dagger} \hat{a}_{2}^{(i)}\right\rangle+1\right),
\end{aligned}
$$

if the two inputs are independent of each other. So a PA is not suitable for mixing photons or quantum information processing with discrete variables. On the other hand, the input-output relations for quadrature-phase amplitudes are given by

$$
\hat{X}_{1,2(\mathrm{PA})}^{(o)}=G \hat{X}_{1,2}^{(i)}+g \hat{X}_{2,1}^{(i)}, \quad \hat{Y}_{1,2(\mathrm{PA})}^{(o)}=G \hat{Y}_{1,2}^{(i)}-g \hat{Y}_{2,1}^{(i)},
$$

which are similar to those for a beam splitter

$$
\hat{X}_{1,2(B S)}^{(o)}=t \hat{X}_{1,2}^{(i)} \pm r \hat{X}_{2,1}^{(i)}, \quad \hat{Y}_{1,2(B S)}^{(o)}=t \hat{Y}_{1,2}^{(i)} \pm r \hat{Y}_{2,1}^{(i)},
$$

where $\hat{X} \equiv \hat{a}+\hat{a}^{\dagger}$ and $\hat{Y} \equiv\left(\hat{a}-\hat{a}^{\dagger}\right) / j(j \equiv \sqrt{-1})$ for the corresponding field described by $\hat{a}$. So they only differ in coupling coefficients. Therefore, for continuous-variable quantum information processing, a PA can play the same role as a BS for superimposing two fields. Note, from Eqs. (4) and (5), that similar to the situation of loss, which introduces quantum noise through the vacuum in the unused port, amplification also adds noise through the vacuum of the second input if it is unattended and thus uncorrelated with the signal input. For this reason, the second input is usually called an idler.

The relationships in Eqs. (4) and (5) provide a way to evaluate quantum state transfer through a BS and a PA, which can be done through Wigner function representation $[14,15]$. For a PA with the input state described by a Wigner function $W_{\text {in }}\left(X_{1}, Y_{1} ; X_{2}, Y_{2}\right)$, the output Wigner function of the PA is given by (for details see the Appendix)

$$
\begin{aligned}
& W_{\text {out }}^{(\mathrm{PA})}\left(x_{1}, y_{1} ; x_{2}, y_{2}\right) \\
& \quad=W_{\text {in }}\left(G x_{1}-g x_{2}, G y_{1}+g y_{2} ; G x_{2}-g x_{1}, G y_{2}+g y_{1}\right) .
\end{aligned}
$$

Similarly for a BS with the same input state, the output state is described by $[14,15]$

$$
\begin{aligned}
& W_{\text {out }}^{(B S)}\left(x_{1}, y_{1} ; x_{2}, y_{2}\right) \\
& \quad=W_{\text {in }}\left(t x_{1}-r x_{2}, t y_{1}-r y_{2} ; t x_{2}+r x_{1}, t y_{2}+r y_{1}\right) .
\end{aligned}
$$

Comparing Eqs. (6) and (7), we find that the output Wigner functions for the two devices give rise to a superposition of input fields but with different phases and different transfer coefficients.
As an example, let us consider the input of a two-mode squeezed state with a Wigner function of [16]

$$
\begin{aligned}
& W_{\text {in }}\left(x_{1}, y_{1} ; x_{2}, y_{2}\right) \\
& =\frac{1}{(2 \pi)^{2}} e^{-\left[\left(x_{1}+x_{2}\right)^{2}+\left(y_{1}-y_{2}\right)^{2}\right] e^{2 s} / 4} \\
& \quad \times e^{-\left[\left(x_{1}-x_{2}\right)^{2}+\left(y_{1}+y_{2}\right)^{2}\right] e^{-2 s} / 4},
\end{aligned}
$$

where $s$ is the squeezing parameter. It is known that when $t=$ $r=1 / \sqrt{2}$, the output of the BS is two single-mode squeezed states with squeezing at orthogonal quadratures. This can be easily confirmed from Eq. (7):

$$
\begin{aligned}
& W_{\text {out }}^{(B S)}\left(x_{1}, y_{1} ; x_{2}, y_{2}\right) \\
& =\frac{1}{(2 \pi)^{2}} e^{-\left(x_{1}^{2}+y_{2}^{2}\right) e^{2 s} / 2} e^{-\left(x_{2}^{2}+y_{1}^{2}\right) e^{-2 s} / 2} \\
& =\frac{1}{2 \pi} e^{-\left(x_{1}^{2} e^{2 s}+y_{1}^{2} e^{-2 s}\right) / 2} \frac{1}{2 \pi} e^{-\left(x_{2}^{2} e^{-2 s}+y_{2}^{2} e^{2 s}\right) / 2} .
\end{aligned}
$$

The corresponding situation for a PA is

$$
\begin{aligned}
W_{\text {out }}^{(\mathrm{PA})}\left(x_{1}, y_{1} ; x_{2}, y_{2}\right) \\
=\frac{1}{(2 \pi)^{2}} e^{-\left[\left(x_{1}+x_{2}\right)^{2}+\left(y_{1}-y_{2}\right)^{2}\right] e^{2 s}(G-g)^{2} / 4} \\
\quad \times e^{\left.-\left[\left(x_{1}-x_{2}\right)^{2}+\left(y_{1}+y_{2}\right)^{2}\right)\right] e^{-2 s}(G+g)^{2} / 4} .
\end{aligned}
$$

Especially when $G+g=1 /(G-g)=e^{s}$, we have

$$
\begin{aligned}
& W_{\text {out }}^{(\mathrm{PA})}\left(x_{1}, y_{1} ; x_{2}, y_{2}\right) \\
& \quad=\frac{1}{2 \pi} e^{-\left(x_{1}^{2}+y_{1}^{2}\right) / 2} \frac{1}{2 \pi} e^{-\left(x_{2}^{2}+y_{2}^{2}\right) / 2},
\end{aligned}
$$

which is just the Wigner function for the vacuum. This is equivalent to the case of balanced gain in an $\mathrm{SU}(1,1)$ interferometer [9].

\section{APPLICATION TO QUANTUM STATE TELEPORTATION}

Consider the scheme shown in Fig. 1 for quantum state teleportation with continuous variables. This is the scheme that makes a complete unconditioned state teleportation (even for the vacuum state) $[4,8]$ in comparison [17] with the teleportation scheme of polarization states [5,7]. In this scheme, an EPR entangled source in the form of Eq. (8) of strength characterized by $s$ is produced from a nondegenerate optical parametric amplifier and its two entangled fields (2 and 3) are sent to Alice and Bob, respectively. A Bell projection measurement is performed with a BS to mix the unknown state (field 1) to be teleported with one of the fields (field 2) of the EPR entangled state, which is described in Eq. (8). The results $\left(i_{X 1^{\prime}}\right.$ and $\left.i_{Y 2^{\prime}}\right)$ of the Bell measurement are sent by Alice via a classical channel to Bob, who modifies field $3\left(M_{X}\right.$ and $\left.M_{Y}\right)$ to recover the original input state characterized by the Wigner function $W_{\text {in }}$.

It has been shown that a parametric amplifier-assisted homodyne measurement can make the same quantum correlation measurement as the homodyne measurement [10]. So let us now replace the BS with a PA of gain parameters $G$ and 


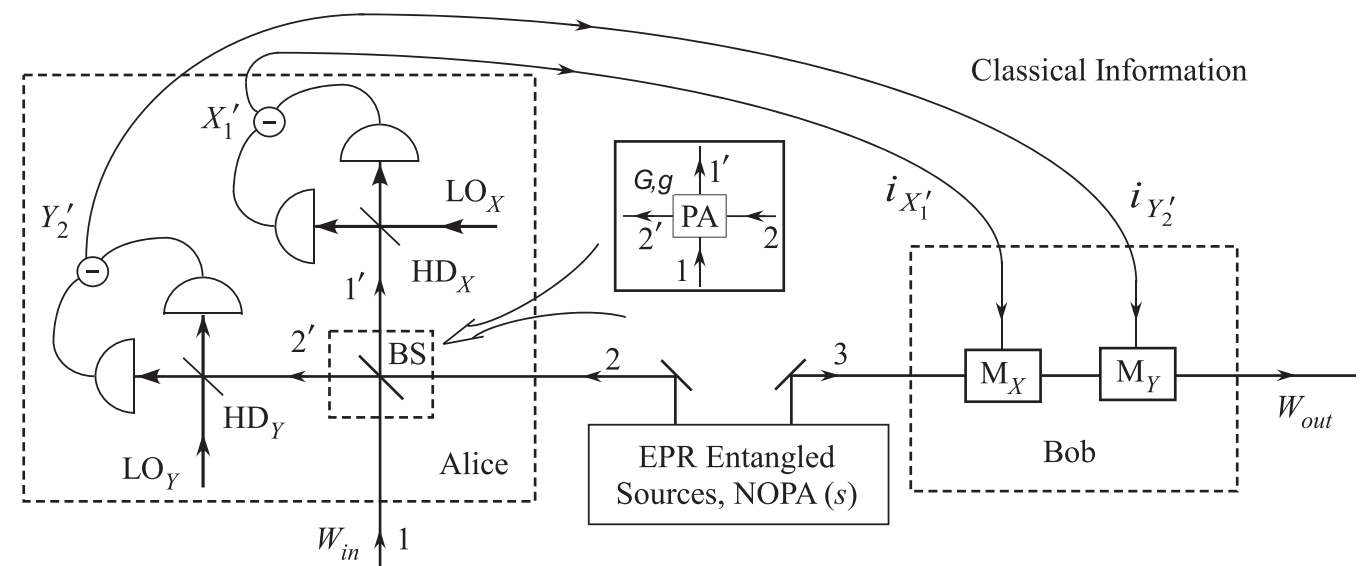

FIG. 1. Schematic of the continuous-variable quantum state teleportation with a parametric amplifier (PA, inset) in place of a beam splitter (BS). Also shown are the nondegenerate optical parametric amplifier (NOPA), homodyne detection (HD), and local oscillator (LO).

$g$, shown in the inset of Fig. 1. Using Eq. (8) for the EPR entangled state with a strength of $s$ and labeling of the fields in Fig. 1, we find that the input state of the parametric amplifier is described by the Wigner function

$$
\begin{aligned}
W_{\text {in }} & \left(x_{1}, y_{1} ; x_{2}, y_{2} ; x_{3}, y_{3}\right) \\
= & \frac{1}{(2 \pi)^{2}} e^{-\left[\left(x_{3}+x_{2}\right)^{2}+\left(y_{3}-y_{2}\right)^{2}\right] e^{2 s} / 4} \\
& \times e^{-\left[\left(x_{3}-x_{2}\right)^{2}+\left(y_{3}+y_{2}\right)^{2}\right] e^{-2 s} / 4} W_{\text {in }}\left(x_{1}, y_{1}\right) .
\end{aligned}
$$

From Eq. (6), the Wigner function after the PA becomes

$$
\begin{aligned}
W_{\text {out }} & \left(x_{1}^{\prime}, y_{1}^{\prime} ; x_{2}^{\prime}, y_{2}^{\prime} ; x_{3}, y_{3}\right) \\
= & \frac{1}{(2 \pi)^{2}} e^{-\left[\left(x_{3}+G x_{2}^{\prime}-g x_{1}^{\prime}\right)^{2}+\left(y_{3}-g y_{1}^{\prime}-G y_{2}^{\prime}\right)^{2}\right] e^{2 s} / 4} \\
& \times e^{-\left[\left(x_{3}-G x_{2}^{\prime}+g x_{1}^{\prime}\right)^{2}+\left(y_{3}+g y_{1}^{\prime}+G y_{2}^{\prime}\right)^{2}\right] e^{-2 s} / 4} \\
& \times W_{\text {in }}\left(G x_{1}^{\prime}-g x_{2}^{\prime}, G y_{1}^{\prime}+g y_{2}^{\prime}\right) .
\end{aligned}
$$

Now we make a homodyne measurement of $\hat{X}_{1^{\prime}}$ and $\hat{Y}_{2^{\prime}}$ of the PA output fields. With a result of $i_{X_{1}^{\prime}}$ and $i_{Y_{2}^{\prime}}$, the state in field 3 (another field of the EPR entangled state) is projected to a state described by the density operator

$$
\hat{\rho}_{\text {proj }}=\operatorname{Tr}_{1^{\prime} 2^{\prime}}\left(\left|i_{X_{1}^{\prime}}, i_{Y_{2}^{\prime}}\right\rangle\left\langle i_{X_{1}^{\prime}}, i_{Y_{2}^{\prime}}\right| \hat{\rho}_{\mathrm{sys}}\right),
$$

where $\left|i_{X_{1}^{\prime}}, i_{Y_{2}^{\prime}}\right\rangle$ is the common eigenstate of $\hat{X}_{1}$ and $\hat{Y}_{2}$. The Wigner function of the projected state is then

$$
\begin{aligned}
& W_{\text {proj }}\left(x_{3}, y_{3}\right) \\
& =\left.\int d x_{2}^{\prime} d y_{1}^{\prime} W_{\text {out }}\left(x_{1}^{\prime}, y_{1}^{\prime} ; x_{2}^{\prime}, y_{2}^{\prime} ; x_{3}, y_{3}\right)\right|_{x_{1}^{\prime}=i_{X_{1}^{\prime}}, y_{2}^{\prime}=i_{Y_{2}^{\prime}}} \\
& =\int \frac{d x_{2}^{\prime} d y_{1}^{\prime}}{(2 \pi)^{2}} e^{-\left[\left(x_{3}+G x_{2}^{\prime}-g i_{X_{1}^{\prime}}\right)^{2}+\left(y_{3}-g y_{1}^{\prime}-G i_{Y_{2}^{\prime}}\right)^{2}\right] e^{2 s} / 4} \\
& \quad \times e^{-\left[\left(x_{3}-G x_{2}^{\prime}+g i_{X_{1}^{\prime}}\right)^{2}+\left(y_{3}+g y_{1}^{\prime}+G i_{Y_{2}^{\prime}}\right)^{2}\right] e^{-2 s} / 4} \\
& \quad \times W_{\text {in }}\left(G i_{X_{1}^{\prime}}-g x_{2}^{\prime}, G y_{1}^{\prime}+g i_{Y_{2}^{\prime}}\right) .
\end{aligned}
$$

Combining the common terms in the integral above, we obtain

$$
\begin{aligned}
& W_{\text {proj }}\left(x_{3}, y_{3}\right) \\
& =\frac{e^{-\left[\left(x_{3}^{2}+y_{3}^{2}\right) / 2 \cosh 2 s\right]}}{(2 \pi)^{2}} \int d x_{2}^{\prime} d y_{1}^{\prime} e^{-(\cosh 2 s / 2)\left(G x_{2}^{\prime}+x_{3} \tanh 2 s-g i_{X_{1}^{\prime}}\right)^{2}}
\end{aligned}
$$

$$
\begin{aligned}
& \times e^{-(\cosh 2 s / 2)\left(g y_{1}^{\prime}+G i_{Y_{2}^{\prime}}-y_{3} \tanh 2 s\right)^{2}} \\
& \times W_{\text {in }}\left(G i_{X_{1}^{\prime}}-g x_{2}^{\prime}, G y_{1}^{\prime}+g i_{Y_{2}^{\prime}}\right) .
\end{aligned}
$$

Taking the limit of $s \gg 1$ so that $\cosh 2 s \gg 1$, we can approximate the Gaussian functions in the integral above with $\delta$ functions. Then we have

$$
\begin{aligned}
W_{\text {proj }}\left(x_{3}, y_{3}\right)= & \frac{1}{2 \pi \cosh 2 s} e^{-(1 / 2 \cosh 2 s)\left(x_{3}^{2}+y_{3}^{2}\right)} \\
& \times \int d x_{2}^{\prime} d y_{1}^{\prime} \delta\left(G x_{2}^{\prime}+x_{3} \tanh 2 s-g i_{X_{1}^{\prime}}\right) \\
& \times \delta\left(g y_{1}^{\prime}+G i_{Y_{2}^{\prime}}-y_{3} \tanh 2 s\right) \\
& \times W_{\text {in }}\left(G i_{X_{1}^{\prime}}-g x_{2}^{\prime}, G y_{1}^{\prime}+g i_{Y_{2}^{\prime}}\right) \\
= & \frac{1}{2 \pi G g \cosh 2 s} e^{-(1 / 2 \cosh 2 s)\left(x_{3}^{2}+y_{3}^{2}\right)} \\
& \times W_{\text {in }}\left(\frac{g}{G} x_{3} \tanh 2 s+\frac{i_{X_{1}^{\prime}}}{G}, \frac{G}{g} y_{3} \tanh 2 s-\frac{i_{Y_{2}^{\prime}}}{g}\right) .
\end{aligned}
$$

When $g \gg 1$ so that $G=\sqrt{1+g^{2}} \approx g$, we have $g / G \approx G / g \approx 1$. Furthermore, if $\sqrt{\cosh 2 s} \gg$ $\max \left\{1\right.$, the range of $\left.W_{\text {in }}\left(x_{3}, y_{3}\right)\right\}$, we have $\tanh 2 s \approx 1$ and $\cosh 2 s \gg x_{3}^{2}+y_{3}^{2}$ so that $e^{-(1 / 2 \cosh 2 s)\left(x_{3}^{2}+y_{3}^{2}\right)} \approx 1$ within the range of $W_{\text {in }}\left(x_{3}, y_{3}\right)$. Then Eq. (17) becomes

$$
\begin{aligned}
& W_{\text {proj }}\left(x_{3}, y_{3}\right) \\
& \simeq \frac{1}{2 \pi G g \cosh 2 s} W_{\text {in }}\left(x_{3}+\frac{i_{X_{1}^{\prime}}}{G}, y_{3}-\frac{i_{Y_{2}^{\prime}}}{g}\right) .
\end{aligned}
$$

The case of finite $G$ and $s$ is dealt with in the Appendix and it is shown that the limits of $G \gg 1$ and $s \gg 1$ are independent. With the detection outcomes of $i_{X_{1}^{\prime}}$ and $i_{Y_{2}^{\prime}}$, we transmit these measurement results through a classical channel to the location of field 3 and a displacement operation of $x_{3}+\frac{i_{X_{1}^{\prime}}}{G} \rightarrow x_{3}$ and $y_{3}-\frac{i_{Y_{2}^{\prime}}}{g} \rightarrow y_{3}\left(\mathrm{M}_{X}\right.$ and $\mathrm{M}_{Y}$ in Fig. 1) can be performed on field 3 , leading to the displaced Wigner function as

$$
W_{\text {proj }}^{\text {disp }}\left(x_{3}, y_{3}\right) \propto W_{\text {in }}\left(x_{3}, y_{3}\right) .
$$


This recovers the Wigner function of the input state, thus achieving quantum state teleportation. So we just showed that the quantum state teleportation scheme still works even after we replace the $\mathrm{BS}$ with a high-gain PA. Note that the condition $G=\sqrt{1+g^{2}} \approx g$ is equivalent to $t=r=1 / \sqrt{2}$, which is required for the scheme with a $\mathrm{BS}$.

\section{TOLERANCE TO DETECTION LOSS}

The quantum teleportation process involves homodyne detection which may introduce losses through detectors' lessthan-unity quantum efficiency and imperfect mode matching with the local oscillator fields. It is known that PA-assisted homodyne measurement is tolerant to detection and propagation losses [10]. We will discuss the influence of these losses in this section. Let us start with the traditional BS scheme. This case was treated in Ref. [4], so we will only present the result here for the comparison with the PA case.
Given the input state in Eq. (12) and using the relation in Eq. (7), we can find the Wigner function after the 50:50 beam splitter of the Bell measurement. We then introduce detection losses by using a beam-splitter model with the same transmissivity $\eta$ for both output fields $1^{\prime}$ and $2^{\prime}$ right before detection. Since the homodyne detection is on the $x$ quadrature of field $1^{\prime}$ and the $y$ quadrature of field $2^{\prime}$, we can leave $y_{1}^{\prime}$ and $x_{2}^{\prime}$ unchanged and integrate them out so that we only consider the effect on $x_{1}^{\prime}$ and $y_{2}^{\prime}$ together with the vacuum from the unused port of the beam splitter: $x_{1}^{\prime} \rightarrow$ $\eta x_{1}^{\prime \prime}+\sqrt{1-\eta^{2}} x_{v 1}^{\prime}, \quad y_{2}^{\prime} \rightarrow \eta y_{2}^{\prime \prime}+\sqrt{1-\eta^{2}} y_{v 2}^{\prime}, \quad x_{v 1} \rightarrow \eta x_{v 1}^{\prime}-$ $\sqrt{1-\eta^{2}} x_{1}^{\prime \prime}$, and $y_{v 2} \rightarrow \eta y_{v 2}^{\prime}-\sqrt{1-\eta^{2}} y_{2}^{\prime \prime}$. Here $\hat{a}_{v 1}^{\prime}$ and $\hat{a}_{v 2}^{\prime}$ are the vacuums coupled in through loss. For the homodyne measurement with a result of $x_{1}^{\prime \prime}=i_{X_{1}^{\prime}}$ and $y_{2}^{\prime \prime}=i_{Y_{2}^{\prime}}$, we obtain the Wigner function for the projected state of field 3 by setting $x_{1}^{\prime \prime}=i_{X_{1}^{\prime}}$ and $y_{2}^{\prime \prime}=i_{Y_{2}^{\prime}}$ and integrating the variables $y_{1}^{\prime}, x_{2}^{\prime}, x_{v 1}^{\prime}$, and $y_{v 2}^{\prime}$. In the limit of $\cosh 2 s \gg 1$, the projected Wigner function is

$$
W_{\text {proj }}^{(B S)}\left(x_{3}, y_{3}\right) \propto \int d x d y W_{\text {in }}(x, y) \exp \left\{-\frac{1}{2 \sigma_{1}^{2}}\left[\left(x-\frac{\sqrt{2} i_{X_{1}^{\prime}}}{\eta}-x_{3}\right)^{2}+\left(y-\frac{\sqrt{2} i_{Y_{2}^{\prime}}}{\eta}-y_{3}\right)^{2}\right]\right\},
$$

where $\sigma_{1}^{2}=2 \frac{1-\eta^{2}}{\eta^{2}}+\frac{1}{\cosh 2 s}$. In the ideal case of no loss, we have $\eta=1$, and for large $s$, the Gaussian in Eq. (20) becomes a $\delta$ function so that after the required displacement of $x_{3}+\frac{\sqrt{2} i_{X_{1}^{\prime}}}{\eta} \rightarrow x_{3}$ and $y_{3}+\frac{\sqrt{2} i_{Y_{2}^{\prime}}}{\eta} \rightarrow y_{3}$ upon receiving the detection outputs $i_{X_{1}^{\prime}}$ and $i_{Y_{2}^{\prime}}$, we recover the input Wigner function $W_{\text {in }}\left(x_{3}, y_{3}\right)$. However, with finite detection losses, vacuum noise will come into the quantum teleportation channel, so even in the limit of large $s$, we have

$$
W_{\text {proj }}^{(B S)}\left(x_{3}, y_{3}\right) \propto \int d x d y W_{\text {in }}(x, y) \exp \left(-\frac{\eta^{2}}{4\left(1-\eta^{2}\right)}\left[\left(x-x_{3}\right)^{2}+\left(y-y_{3}\right)^{2}\right]\right),
$$

which involves a convolution with the vacuum Wigner function. Equation (21) was first derived in Ref. [4].

For the scheme with a PA in place of the BS, we introduce losses after the output of the PA but before the homodyne measurement. From Eq. (16) and a procedure similar to Eq. (20) but without the cosh $2 s \gg 1$ assumption, we have the projected Wigner function of field 3 after the homodyne measurement of results $x_{1}^{\prime}=i_{X_{1}^{\prime}}$ and $y_{2}^{\prime}=i_{Y_{2}^{\prime}}$ :

$$
\begin{aligned}
W_{\text {proj }}^{(\mathrm{PA})}\left(x_{3}, y_{3}\right)= & \frac{1}{(2 \pi)^{3}} e^{-(1 / 2 \cosh 2 s)\left(x_{3}^{2}+y_{3}^{2}\right)} \int d x_{2}^{\prime} d y_{1}^{\prime} d x_{v 1}^{\prime} d y_{v 2}^{\prime} \exp \left(-\frac{\cosh 2 s}{2}\left[G x_{2}^{\prime}+x_{3} \tanh 2 s-g\left(\eta i_{X_{1}^{\prime}}+\sqrt{1-\eta^{2}} x_{v 1}^{\prime}\right)\right]^{2}\right) \\
& \times \exp \left(-\frac{\cosh 2 s}{2}\left[g y_{1}^{\prime}+G\left(\eta i_{Y_{2}^{\prime}}+\sqrt{1-\eta^{2}} y_{v 2}^{\prime}\right)-y_{3} \tanh 2 s\right]^{2}\right) \\
& \times \exp \left(-\frac{1}{2}\left[\left(\eta x_{v 1}^{\prime}-\sqrt{1-\eta^{2}} i_{X_{1}^{\prime}}\right)^{2}+\left(\eta y_{v 2}^{\prime}-\sqrt{1-\eta^{2}} i_{Y_{2}^{\prime}}\right)^{2}\right]\right) \\
& \times W_{\text {in }}\left(G\left(\eta i_{X_{1}^{\prime}}+\sqrt{1-\eta^{2}} x_{v 1}^{\prime}\right)-g x_{2}^{\prime}, G y_{1}^{\prime}+g\left(\eta i_{Y_{2}^{\prime}}+\sqrt{1-\eta^{2}} y_{v 2}^{\prime}\right)\right) .
\end{aligned}
$$

Now we make a change of integral variables from $x_{2}^{\prime}$ and $y_{1}^{\prime}$ to $x=G\left(\eta i_{X_{1}^{\prime}}+\sqrt{1-\eta^{2}} x_{v 1}^{\prime}\right)-g x_{2}^{\prime}$ and $y=G y_{1}^{\prime}+g\left(\eta i_{Y_{2}^{\prime}}+\right.$ $\left.\sqrt{1-\eta^{2}} y_{v 2}^{\prime}\right)$. After integrating over $x_{v 1}^{\prime}$ and $y_{v 2}^{\prime}$, we obtain the projected Wigner function in the form of

$$
\begin{aligned}
W_{\text {proj }}^{(\mathrm{PA})}\left(x_{3}, y_{3}\right) \propto & e^{-(1 / 2 \cosh 2 s)\left(x_{3}^{2}+y_{3}^{2}\right)} \int d x d y W_{\text {in }}(x, y) \exp \left[-\frac{1}{2 \sigma_{2 x}^{2}}\left(x-\frac{i_{X_{1}^{\prime}}}{\eta G}-\frac{g x_{3}}{G} \tanh 2 s\right)^{2}\right] \\
& \times \exp \left[-\frac{1}{2 \sigma_{2 y}^{2}}\left(y+\frac{i_{Y_{2}^{\prime}}}{\eta g}-\frac{G y_{3}}{g} \tanh 2 s\right)^{2}\right],
\end{aligned}
$$

where $\sigma_{2 x}^{2}=\frac{1-\eta^{2}}{\eta^{2} G^{2}}+\frac{g^{2}}{G^{2} \cosh 2 s}$ and $\sigma_{2 y}^{2}=\frac{1-\eta^{2}}{\eta^{2} g^{2}}+\frac{G^{2}}{g^{2} \cosh 2 s}$. Setting $G \gg 1$ and $s \gg 1$ so that $G \approx g$ and tanh $2 s \approx 1$ and $\cosh 2 s \gg$ 1 and making the displacement operation of $x_{3}+i_{X_{1}^{\prime}} / \eta g \rightarrow x_{3}$ and $y_{3}-i_{Y_{2}^{\prime}} / \eta G \rightarrow y_{3}$, we have

$$
W_{\text {proj }}^{(\mathrm{PA})}\left(x_{3}, y_{3}\right) \propto \int d x d y W_{\text {in }}(x, y) e^{-\left(1 / 2 \sigma_{2}^{2}\right)\left[\left(x-x_{3}\right)^{2}+\left(y-y_{3}\right)^{2}\right]},
$$


where $\sigma_{2}^{2} \equiv \frac{1-\eta^{2}}{\eta^{2} G^{2}}+\frac{1}{\cosh 2 s} \approx \sigma_{2 x}^{2} \approx \sigma_{2 y}^{2}$. Notice that the conditions of $G \approx g$ and $s \gg 1$ are independent of each other (discussed further in the Appendix). Comparing $\sigma_{2}^{2}$ with $\sigma_{1}^{2}$ in Eq. (20) for the BS scheme, we find that the PA scheme reduces the effect of losses by a factor of $2 G^{2}$. If $G^{2} \sim$ $\left(1-\eta^{2}\right) e^{2 s} / 2 \eta^{2}$, we have $\sigma_{2}^{\prime 2} \rightarrow 4 e^{-2 s}$, which is similar to the lossless case $(\eta=1)$ of Eq. (20). Therefore, the effect of losses can be mitigated by large $G$.

\section{INFLUENCE OF LOSSES VIA FIDELITY}

To quantify the influence of losses and the gain size of a PA in place of the BS, we consider the fidelity of teleportation. For a pure input state $\left|\phi_{\text {in }}\right\rangle$ and an output described by density operator $\hat{\rho}_{\text {out }}$, it is given by [5]

$$
F=\left\langle\phi_{\text {in }}\left|\hat{\rho}_{\text {out }}\right| \phi_{\text {in }}\right\rangle .
$$

Note that if the output state is the same as the input, $\hat{\rho}_{\text {out }}=$ $\left|\phi_{\text {in }}\right\rangle\left\langle\phi_{\text {in }}\right|$, Eq. (25) gives $F=1$.

Since the roles of $G$ and $s$ are independent of each other, for the simplicity of argument and emphasis on the effect of the PA, we set $s \rightarrow \infty$ here. The case of finite $s$ will be presented in the Appendix and the conclusion is similar to the case of $s \rightarrow \infty$.

\section{A. Scheme with a beam splitter}

Consider the quantum state teleportation scheme as the quantum channel. For the case of using a BS for a Bell measurement and with large $s$, the Wigner function of the output is connected to the input by Eq. (21) and is rewritten as

$$
W_{\text {out }}=W_{\text {in }} \circ G_{\bar{\sigma}_{1}},
$$

with $\circ$ denoting convolution and $G_{\bar{\sigma}_{1}}$ the two-dimensional Gaussian distribution with a variance $\bar{\sigma}_{1}^{2}=2\left(1-\eta^{2}\right) / \eta^{2}$. The teleportation input-output relation for the Wigner functions described by Eq. (26) can also be cast in the density operator form as (see the Appendix)

$$
\hat{\rho}_{\text {out }}=\int d x d y \hat{D}\left(\frac{x+y j}{2}\right) \hat{\rho}_{\text {in }} \hat{D}^{\dagger}\left(\frac{x+y j}{2}\right) G_{\bar{\sigma}_{1}}(x, y),
$$

where the operator $\hat{D}(\alpha) \equiv \exp \left(\alpha \hat{a}^{\dagger}-\alpha^{*} \hat{a}\right)$ is the displacement operator $[\alpha=(x+j y) / 2]$.

From Eq. (25) we find that the fidelity for a pure state input is then

$$
F=\int d x d y\left|\chi_{\text {in }}(x, y)\right|^{2} G_{\bar{\sigma}_{1}}(x, y)
$$

where $\chi_{\text {in }}(x, y)=\left\langle\phi_{\text {in }}\left|\hat{D}\left(\frac{x+y j}{2}\right)\right| \phi_{\text {in }}\right\rangle$, which is the single-mode characteristic function defined in Eq. (A1) for the input state's Wigner function. We can now evaluate the fidelity for a number of known input states. First, for the coherent state, it is easy to obtain the fidelity as

$$
F=\frac{1}{1+\bar{\sigma}_{1}^{2} / 2},
$$

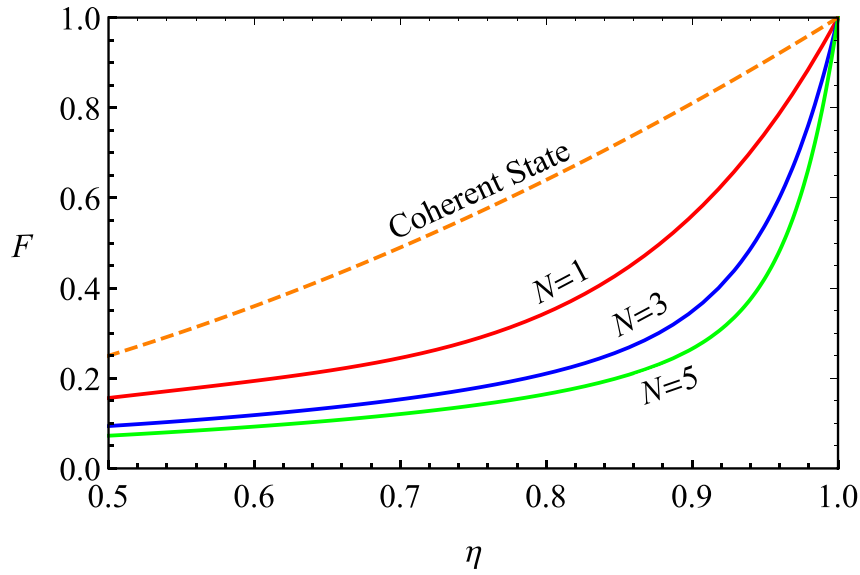

FIG. 2. Fidelity $F$ as a function of detection loss in the teleportation scheme with a BS for a coherent state $|\alpha\rangle$ with $\alpha=3+3 j$ (dashed line) and Fock states of $N=1,3,5$.

with $\bar{\sigma}_{1}^{2} \equiv 2\left(1-\eta^{2}\right) / \eta^{2}$. For the Fock state $|N\rangle$,

$$
\begin{aligned}
\left|\chi_{\text {in }}(x, y)\right|^{2} & =\left|\left\langle N\left|\hat{D}\left(\frac{x+y j}{2}\right)\right| N\right\rangle\right|^{2} \\
& =\left|\int d x^{\prime} f_{N}^{*}\left(x^{\prime}\right) f_{N}\left(x^{\prime}-x / \sqrt{2}\right) e^{j\left[y\left(x^{\prime}-x / \sqrt{2}\right) / \sqrt{2}\right]}\right|^{2},
\end{aligned}
$$

where

$$
f_{N}(x)=\frac{\pi^{-1 / 4}}{\sqrt{2^{N} N !}} e^{-x^{2} / 2} H_{N}(x),
$$

with $H_{N}(x)$ the $N$ th-order Hermite polynomials. We can then evaluate numerically the fidelity $F$ with Eq. (28) for a given loss modeled by a BS with transmission coefficient $\eta$. Figure 2 plots the dependence of $F$ as a function of $\eta$ for a coherent state of $\alpha=3+3 j$ and Fock states of $N=1,3,5$, showing a fast drop of $F$ with the increase of loss (decrease of $\eta$ ). The rate of drop is especially large for number states with higher photon numbers as compared to the coherent state (dashed curve). Thus, nonclassical states are more sensitive to loss in the teleportation process.

\section{B. Scheme with a parametric amplifier}

Next we consider the teleportation scheme with the aid of a PA but having losses $1-\eta$ before detection. The output is related to input by Eq. (23) but with $s \gg 1$ and can be rewritten in the form of Eq. (26) as

$$
W_{\text {out }}(x, y)=\int_{\times G_{\bar{\sigma}_{2 x}}\left(x^{\prime}\right) G_{\bar{\sigma}_{2 y}}\left(y^{\prime}\right),} d x^{\prime} d y^{\prime} W_{\text {in }}\left(x \frac{g}{g}-x^{\prime}, y \frac{G}{g}-y^{\prime}\right)
$$

where $\bar{\sigma}_{2 x}^{2} \equiv\left(1-\eta^{2}\right) / \eta^{2} G^{2}$ and $\bar{\sigma}_{2 y}^{2} \equiv\left(1-\eta^{2}\right) / \eta^{2} g^{2}$. Then Eq. (27) is changed to (see the Appendix)

$$
\hat{\rho}_{\text {out }}=\int_{\times \hat{S}^{\dagger}(\epsilon) G_{\bar{\sigma}_{2 x}}(x) G_{\bar{\sigma}_{2 y}}(y),}
$$




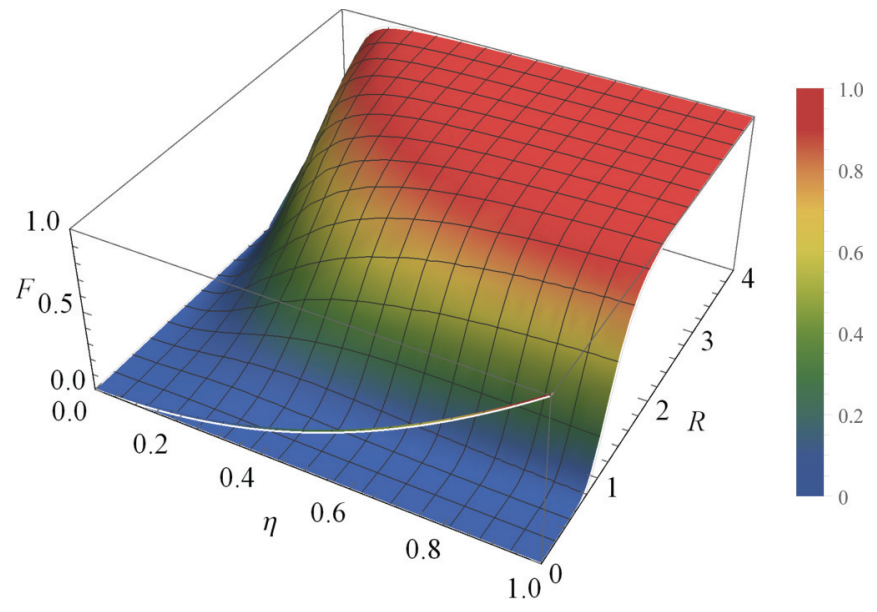

FIG. 3. Three-dimensional plot of fidelity $F$ as a function of transmission $\eta$ (opposite of loss) and the gain parameter $R(G \equiv$ $\cosh R$ ) of the parametric amplifier used in the Bell measurement for teleportation of the coherent state $|\alpha\rangle$ with $\alpha=3+3 j$. The light curve at $R=0$ corresponds to the case of using a beam splitter for the Bell measurement (the dashed line in Fig. 2).

where $\hat{S}(\epsilon) \equiv \exp \left[\epsilon\left(\hat{a}^{\dagger 2}-\hat{a}^{2}\right) / 2\right]$ is the squeezing operator with $\epsilon \equiv \ln (G / g)$, and Eq. (28) is modified to

$$
F=\int d x d y\left|\chi_{P A}(x, y)\right|^{2} G_{\bar{\sigma}_{2 x}}(x) G_{\bar{\sigma}_{2 y}}(y),
$$

with $\chi_{P A}(x, y)=\left\langle\phi_{\text {in }}\left|\hat{S}(\epsilon) \hat{D}\left(\frac{x+y j}{2}\right)\right| \phi_{\text {in }}\right\rangle$.

For a coherent state $|\alpha\rangle$, we have

$$
\begin{aligned}
\left|\chi_{P A}^{(\alpha)}(x, y)\right|^{2} & =\left|\left\langle\alpha\left|\hat{S}(\epsilon) \hat{D}\left(\frac{x+y j}{2}\right)\right| \alpha\right\rangle\right|^{2} \\
& =\left|\left\langle\alpha|\hat{S}(\epsilon)| \alpha+\frac{x+y j}{2}\right\rangle\right|^{2} .
\end{aligned}
$$

Setting $\alpha=a+b j$, we then obtain from Eq. (34), with some manipulation,

$$
\begin{aligned}
F & =\frac{2 \exp \left(-\frac{2 a^{2}(1-g / G)^{2}}{1+g^{2} / G^{2}+\bar{\sigma}_{2 x}^{2}}-\frac{2 b^{2}(1-G / g)^{2}}{1+G^{2} / g^{2}+\bar{\sigma}_{2 y}^{2}}\right)}{\sqrt{\left(1+g^{2} / G^{2}+\bar{\sigma}_{2 x}^{2}\right)\left(1+G^{2} / g^{2}+\bar{\sigma}_{2 y}^{2}\right)}} \\
& \approx \frac{\exp \left(-\frac{1}{4 G^{4}} \frac{a^{2}+b^{2}}{1+\bar{\sigma}_{2}^{2} / 2}\right)}{1+\bar{\sigma}_{2}^{2} / 2} \text { for } G \gg 1,
\end{aligned}
$$

where $\bar{\sigma}_{2}^{2} \equiv\left(1-\eta^{2}\right) / \eta^{2} G^{2}$. When $G$ tends to a large value, we have $G \sim g$ and Eq. (36) approaches Eq. (29) but with $\bar{\sigma}_{1}^{2}$ replaced by $\bar{\sigma}_{2}^{2}$, which goes to zero as $G$ becomes large. Hence, $F \rightarrow 1$ for large $G$ and independent of the loss $\eta$. So with the aid of a PA of large gain, the effect of detection loss can be reduced to zero. This is demonstrated in Fig. 3 as the red region $(F \sim 1)$ in the three-dimensional plot of $F$ as a function of $\eta$ and the gain-related parameter $R$ with $G \equiv \cosh R$ [or $\left.R \equiv \ln \left(G+\sqrt{G^{2}-1}\right)\right]$. Figure 3 is obtained from the first expression in Eq. (36) without approximation. The red region extends to low values of $\eta(<0.5$, large loss) at high gain $(R>2)$. The light colored curve at $R=0$ is for the case when we use a beam splitter for the Bell measurement (the dashed line in Fig. 2). In this case, as can be seen, $F$ drops fast as $\eta$ decreases.

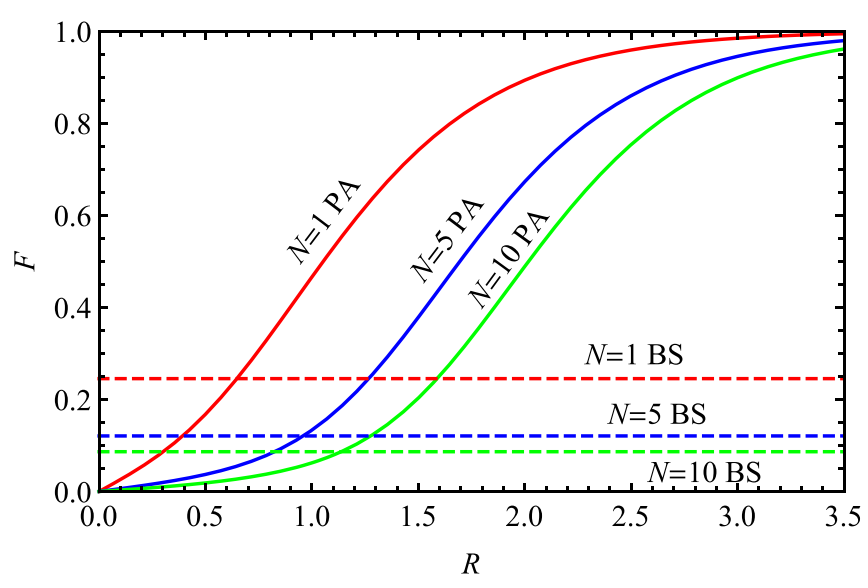

FIG. 4. Fidelity $F$ as a function of gain of parametric amplifier at a loss of $\eta=0.7$ for the Fock state $|N\rangle$ with $N=1,5,10$. The dashed lines are for the BS scheme.

On the other hand, even with no detection loss $(\eta=1$ and $\bar{\sigma}_{2 x}=\bar{\sigma}_{2 y}=0$ ) but a finite $G$, we have, from Eq. (36),

$$
\begin{aligned}
F & =\frac{2 G g}{G^{2}+g^{2}} \exp \left(-2 \frac{(G-g)^{2}}{G^{2}+g^{2}}\left(a^{2}+b^{2}\right)\right) \\
& \approx \exp \left[-\left(a^{2}+b^{2}\right) / 4 G^{4}\right] \quad \text { for } G \gg 1 .
\end{aligned}
$$

The blue region (low $F<0.15$ ) in Fig. 3 extends to high- $\eta$ values when $R<1$ for relatively low gain, which indicates that high gain $(R>2)$ is required for the PA-assisted scheme. From Eq. (37) we find that in order to have $F \approx 1$, we need $G^{2} \gg \sqrt{a^{2}+b^{2}}=|\alpha|$, that is, the larger the average photon number is, the bigger the gain $G$ needs to be. This behavior is not limited to coherent states, as we will see next for photon number Fock states.

Next we look at the nonclassical states of the Fock state $|N\rangle$. The characteristic function $\chi_{P A}$ in Eq. (34) has the form of

$$
\begin{aligned}
\left|\chi_{P A}^{(N)}(x, y)\right|^{2}= & \left.\left\langle N\left|\hat{S}(\epsilon) \hat{D}\left(\frac{x+y j}{2}\right)\right| N\right\rangle\right|^{2} \\
= & \mid \int d x^{\prime} \sqrt{\tanh R} e^{-j y\left[\left(x^{\prime} / \sqrt{2}\right) \tanh (\epsilon)+x / 2\right]} \\
& \times\left. f_{N}^{*}\left(x^{\prime}\right) f_{N}\left(-x^{\prime} \tanh (\epsilon)-x / \sqrt{2}\right)\right|^{2},
\end{aligned}
$$

where the definition of $f_{N}(x)$ is the same as in Eq. (31). The fidelity can be calculated numerically from Eq. (34). We plot in Fig. 4 the fidelity $F$ as a function of the gain-related parameter $R$ for Fock states $|N\rangle$, with $N=1,5,10$, respectively. The detection loss is set with transmission $\eta=0.7$. As can be seen, larger gain ( $R$ value) is needed for higher $N$ to reach $F \approx 1$, similarly to the case of coherent states as predicted by the second line of Eq. (37). We also plot in Fig. 4 the corresponding values of $F$ for the BS scheme (dashed lines) for comparison, demonstrating the effect of the PA to counter the detrimental effect of detection loss. The effect of loss on the Fock state $|5\rangle$ is displayed in Fig. 5, where we plot $F$ as a function of transmission coefficient $\eta$ for $R=1,2,3$ ( $G=$ $1.54,3.76,10.07)$, respectively. The result of the BS scheme (dashed line) is also plotted for comparison. As expected, the 


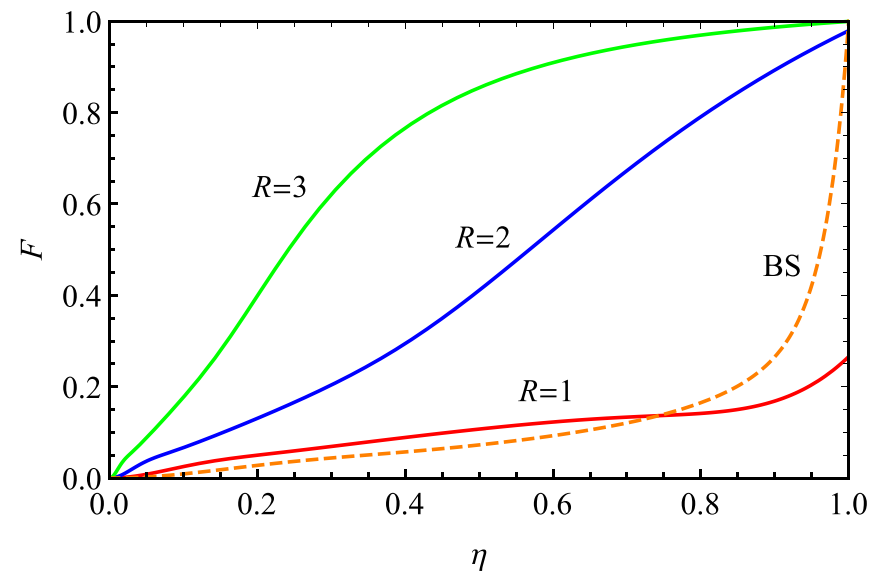

FIG. 5. Fidelity $F$ as a function of transmission $\eta$ for the Fock state $|N\rangle$ with $N=5$ at a gain of $R=1,2,3(G=1.54,3.76,10.07)$ for the PA-assisted scheme. The case of the BS scheme is plotted as a dashed line for comparison.

PA-assisted scheme is not good for the case of relatively low gain $(R=1,2)$, but with $R=3$, it keeps a relatively high $F$ value (greater than 0.8$)$ even at a large loss of $50 \%(\eta=0.5)$.

\section{INFLUENCE OF LOSS ON ENTANGLEMENT}

The input states in the previous sections are all pure states. In quantum communication, we more often transmit one field of entangled fields, as in, for example, the entanglement swapping protocol [18-20]. The transmitted field is usually in a mixed state when the other entangled field is not considered. We will examine how losses in the two teleportation schemes will affect the transmission of an EPR type of entangled state which is simply a two-mode squeezed state with a Wigner function given in Eq. (8).

\section{A. Fidelity}

For an input field that is entangled with another field, after tracing out the other field, it becomes a mixed state described by density operator $\hat{\rho}_{\text {in }}$. In this case, we cannot use Eq. (25) or its density operator form $F^{\prime}=\operatorname{Tr}\left(\hat{\rho}_{\text {in }} \hat{\rho}_{\text {out }}\right)$, for even in the ideal transmitted case of $\hat{\rho}_{\text {out }}=\hat{\rho}_{\text {in }}, F^{\prime}=\operatorname{Tr}\left(\hat{\rho}_{\text {in }}^{2}\right) \neq 1$ for mixed states. A widely used definition of fidelity for mixed states was given by Jozsa as $F_{m}=\operatorname{Tr}\left[\sqrt{\sqrt{\hat{\rho}_{\text {in }}} \hat{\rho}_{\text {out }} \sqrt{\hat{\rho}_{\text {in }}}}\right]^{2}$ [21], but this definition does not consider entanglement with the other field.

On the other hand, we can use the entanglement fidelity [22], which quantifies how well a quantum (teleportation) channel, which may interact with the environment $E$, preserves the transferred input state (in a state space denoted by $Q)$ and its entanglement with another system in the space of $R$. The input state $\left(\hat{\rho}_{\text {in }}\right)$ can be obtained by taking the partial trace of an entangled pure state on a larger Hilbert space (a joint space of $Q$ and the entangled space $R$ ) over the space $R$. For the case of no entanglement, the input state is then a pure state. According to Ref. [22], the entanglement fidelity only depends on the initial quantum state and the dynamic evolution of the input state on $Q$ through the quantum channel. Suppose the general quantum evolution of the state on
$Q$ through the quantum channel can be cast in the form of $\hat{\rho}_{\text {out }}=\sum_{k} \hat{A}_{k} \hat{\rho}_{\text {in }} \hat{A}_{k}^{\dagger}$ by some operator-sum representation with $\hat{A}_{i}$ being a collection of operators acting in the space of $Q$ and satisfying the completeness relation $\sum_{k} \hat{A}_{k}^{\dagger} \hat{A}_{k}=1$. Then entanglement fidelity is defined as [22]

$$
F_{e}=\sum_{k} \operatorname{Tr}\left(\hat{A}_{k} \hat{\rho}_{\text {in }}\right) \operatorname{Tr}\left(\hat{A}_{k}^{\dagger} \hat{\rho}_{\text {in }}\right) .
$$

For the special case of a pure input state $\hat{\rho}_{\text {in }}=\left|\phi_{\text {in }}\right\rangle\left\langle\phi_{\text {in }}\right|$, we have $F_{e}=\sum_{k}\left|\left\langle\phi_{\text {in }}\left|\hat{A}_{k}\right| \phi_{\text {in }}\right\rangle\right|^{2}=\left\langle\phi_{\text {in }}\left|\hat{\rho}_{\text {out }}\right| \phi_{\text {in }}\right\rangle$, which recovers Eq. (25).

In our derivation of the output Wigner function for both teleportation protocols, the output density operators of Eqs. (27) and (33) have already been written in the form of $\hat{\rho}_{\text {out }}=\sum_{k} \hat{A}_{k} \hat{\rho}_{\text {in }} \hat{A}_{k}^{\dagger}$, with $\hat{A}_{k} \equiv \hat{D}\left(\frac{x+j y}{2}\right)$ and $\hat{S}(\epsilon) \hat{D}\left(\frac{x+j y}{2}\right)$ for the BS and PA schemes, respectively. Note that the sum over $k$ is replaced by the integral over $x$ and $y$.

To obtain $\hat{\rho}_{\text {in }}$ for Eq. (39), we just need to take the partial trace of the other field component of the density operator of the entangled states. For the EPR state (a two-mode squeezed state) in Eq. (8), the signal field becomes a thermal state with an average photon number $\bar{n}=\sinh ^{2}(s)$. Its density operator can be expressed by the $P$ representation as $\hat{\rho}_{\text {in }}=\int d^{2} \alpha P(\alpha)|\alpha\rangle\langle\alpha|$, where $P(\alpha)=\frac{1}{\pi \bar{n}} e^{-|\alpha|^{2} / \bar{n}}$. The entanglement fidelity $F_{e}$ can be obtained from Eqs. (28) and (34) with the characteristic functions being

$$
\begin{aligned}
\left|\chi_{B S}(x, y)\right|^{2}=\left|\int d^{2} \alpha P(\alpha)\left\langle\alpha\left|\hat{D}\left(\frac{x+y j}{2}\right)\right| \alpha\right\rangle\right|^{2} \\
=\mid \int d^{2} \alpha \frac{1}{\pi \bar{n}} e^{-|\alpha|^{2} / \bar{n}} \\
\times\left. e^{\left[(x+y j) \alpha^{*}-(x-y j) \alpha\right] / 2-\left(x^{2}+y^{2}\right) / 8}\right|^{2}
\end{aligned}
$$

for the BS scheme and

$$
\begin{aligned}
&\left|\chi_{P A}(x, y)\right|^{2}=\mid \int\left.d^{2} \alpha P(\alpha)\left\langle\alpha\left|\hat{S}(\epsilon) \hat{D}\left(\frac{x+y j}{2}\right)\right| \alpha\right\rangle\right|^{2} \\
&=\mid \int d^{2} \alpha \frac{1}{\pi \bar{n}} e^{-|\alpha|^{2} / \bar{n}} e^{\left[(x+y j) \alpha^{*}-(x-y j) \alpha\right] / 4} \\
& \times\left.\left\langle\alpha|\hat{S}(\epsilon)| \alpha+\frac{x+y j}{2}\right\rangle\right|^{2}
\end{aligned}
$$

for the PA-assisted scheme. Figure 6 shows the results of the calculation. It is very similar to Fig. 5 for the number state case.

\section{B. Inseparability}

Another way to see how entanglement is affected by teleportation protocols is to check the inseparability criterion through the parameter $I_{S}$ defined as [23]

$$
I_{s} \equiv\left\langle\Delta^{2}\left(\hat{X}_{1}-\hat{X}_{2}\right)\right\rangle+\left\langle\Delta^{2}\left(\hat{Y}_{1}+\hat{Y}_{2}\right)\right\rangle .
$$

For unentangled fields, it has a minimum value of $I_{s}^{(0)}=4$ for the vacuum. Here $I_{s}<I_{s}^{(0)}=4$ gives the criterion for entanglement between two fields and the smaller the value of $I_{s}$ is, the more entangled the two fields are. The ideal value is $I_{s}=0$, showing perfect EPR correlation between $\hat{X}_{1}$ and $\hat{X}_{2}$ and between $\hat{Y}_{1}$ and $\hat{Y}_{2}$. For the EPR entangled state given in Eq. (8) with $s=-1$, we have the normalized value 


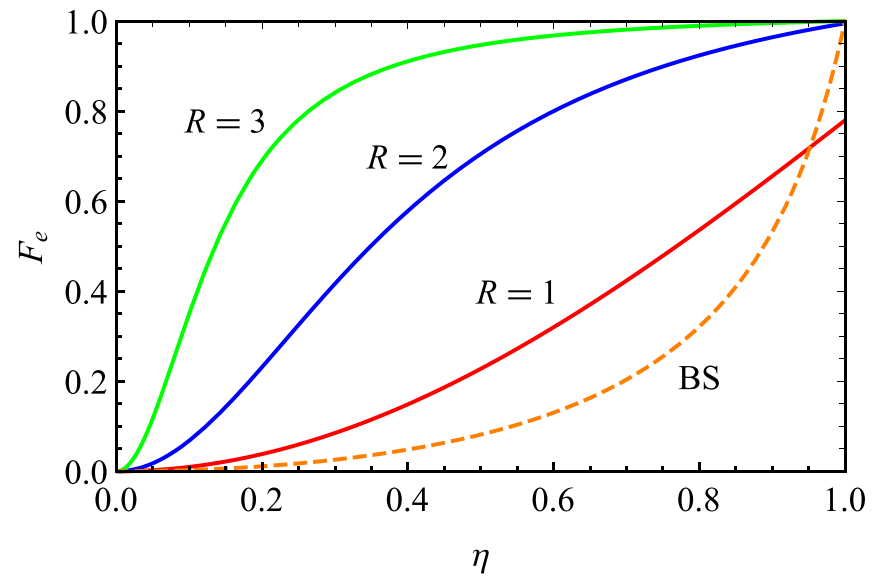

FIG. 6. Entanglement fidelity $F_{e}$ as a function of transmission $\eta$ for a thermal state (one field of the EPR-entangled two-mode squeezed state) with average photon number $\bar{n}=\sinh ^{2}(-1)=1.38$ for the PA-assisted scheme with gain parameters of $R=1,2,3$ (solid lines) and for the BS scheme (dashed line).

$I_{s}^{\mathrm{EPR}} / I_{s}^{(0)}=0.135=-8.69 \mathrm{~dB}$. We will teleport one of the two entangled fields, say, the signal beam, through the BS or PA-assisted teleportation scheme.

The Wigner functions of the output state are given by Eqs. (26) and (32) as

$$
\begin{aligned}
W_{\text {out }}= & \int_{\times G_{\sigma_{x}^{\prime}}\left(x^{\prime}\right) G_{\sigma_{y}^{\prime}}\left(y^{\prime}\right),} d x^{\prime} d y^{\prime} W_{\text {in }}\left(x_{1}, y_{1} ; x_{2}^{\prime}-x^{\prime}, y_{2}^{\prime}-y^{\prime}\right) \\
& \text {, }
\end{aligned}
$$

with $x_{2}^{\prime}=x_{2}, y_{2}^{\prime}=y_{2}$, and $\sigma_{x}^{\prime}=\sigma_{y}^{\prime}=\bar{\sigma}_{1}$ for the BS scheme and $x_{2}^{\prime}=x_{2} \frac{g}{G}, y_{2}^{\prime}=y_{2} \frac{G}{g}, \sigma_{x}^{\prime}=\bar{\sigma}_{2 x}$, and $\sigma_{y}^{\prime}=\bar{\sigma}_{2 y}$ for the PAassisted scheme. We calculate $I_{s}^{\text {out }}$ between the teleported signal field and the original idler field to examine how entanglement is affected by teleportation. Further, $I_{s}^{\text {out }}=$ $\left\langle\Delta^{2} X_{-}\right\rangle_{\text {out }}+\left\langle\Delta^{2} Y_{+}\right\rangle_{\text {out }}$ with $X_{-}=x_{1}-x_{2}$ and $Y_{+}=y_{1}+y_{2}$ is calculated from the Wigner function by

$$
\langle A\rangle_{\text {out }}=\int_{\times W_{\text {out }}\left(x_{1}, y_{1} ; x_{2}, y_{2}\right),} d x_{1} d y_{1} d x_{2} d y_{2} A\left(x_{1}, y_{1} ; x_{2}, y_{2}\right)
$$

where $A=\Delta^{2} X_{-}$and $\Delta^{2} Y_{+}$, respectively. The $W_{\text {out }}$ is obtained from Eq. (43) with $W_{\text {in }}$ given in Eq. (8) for an EPR entangled state. Figure 7 shows the results of calculation. As can be seen, the BS scheme (dashed curve) is very sensitive to losses: The value of $I_{s}$ increases quickly as detection efficiency $\eta$ drops and the fields are unentangled for $\eta<0.7$ (the thin gray line at $0 \mathrm{~dB}$ is the threshold for entanglement). The PA-assisted scheme, on the other hand, can keep $I_{s}$ at quite a low value with a large gain $(R=3)$ even for $\eta$ as low as 0.5 . A small gain cannot preserve the original $I_{s}$ value even at no loss $\eta=1$, but the fields are still entangled up to $\eta=0.5$.

Comparing Figs. 6 and 7, we find that there is a high correlation between entanglement fidelity $F_{e}$ and inseparability $I_{s}$, although there is no direct connection between the two quantities. This reflects that $F_{e}$ indeed quantifies how well the entanglement is preserved through transmission. In some sense, they both provide a general characterization of the transmission quality of the teleportation protocols.

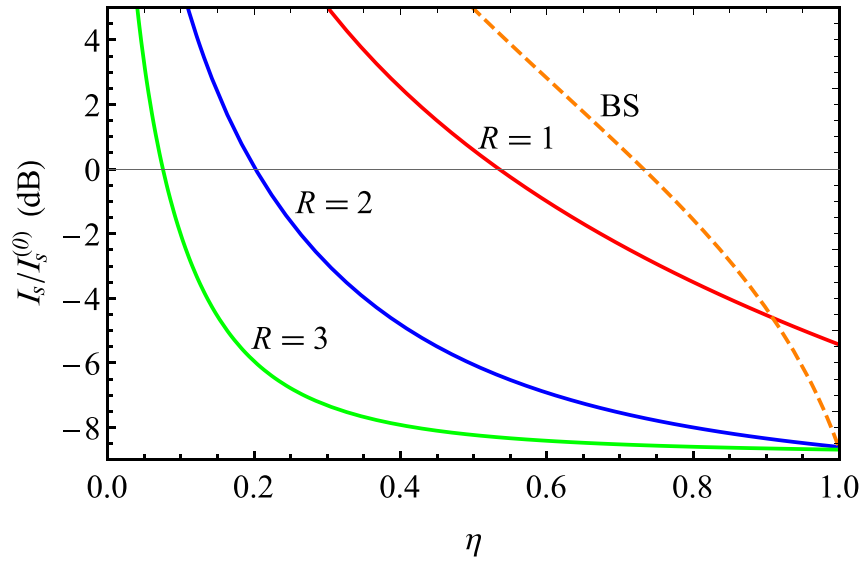

FIG. 7. Normalized inseparability $I_{s} / I_{s}^{(0)}\left(I_{s}^{(0)}=4\right.$ for the vacuum) in logarithmic scale as a function of transmission $\eta$ for the EPR-entangled state with initial input $I_{s}^{\mathrm{EPR}} / I_{s}^{(0)}=0.135=$ $-8.69 \mathrm{~dB}$ for various gain parameters of $R=1,2,3$ for the PAassisted scheme (solid lines) and the BS scheme (dashed line). The light black line at $0 \mathrm{~dB}$ is the threshold for entanglement.

\section{CONCLUSION}

In summary, we have studied the quantum state teleportation scheme with a parametric amplifier replacing the beam splitter for field mixing used in the Bell measurement process. With large enough gain for the PA, the scheme is as good as the original scheme. In addition to field mixing, the PA amplifies the input field to a level that is much higher than the vacuum quantum noise so that it can overcome the detection loss in the Bell measurement process, leading to a high teleportation fidelity even in the presence of a large detection loss. This will be especially useful for the spectrum of light where no efficient detector is available. However, internal losses of the PA and the losses before the PA such as mode mismatch will be the losses imposed on the incoming fields before the PA and thus cannot be overcome by the employment of the PA [9]. They will have the same effect as in the BS scheme.

The addition of an active element of the PA to replace a passive element like a BS will not only require extra resources in the pumping of the PA but also add an extra level of control such as locking of the pump phase to the EPR source. In a real experiment, these can be easily handled, as shown in a recent demonstration in a fiber system [10] where a PA is used to replace a BS in battling the large coupling losses in fibers. The requirement of $R>2$ discussed in Sec. $\mathrm{V}$ for a good fidelity corresponds to $G>3.8$ or a power gain larger than 15 , which can be easily achieved experimentally [10]. On the other hand, a larger average photon number requires a higher gain, as shown in Eq. (37) and in Figs. 4 and 5. However, states with a larger photon number are more prone to losses in the BS scheme. So one must balance between the cost and benefit of loss tolerance in selecting a PA.

The application of a PA is not limited to quantum teleportation schemes. The general principle of loss tolerance is applicable to overcome any loss before detection. For example, there may exist large losses in coupling light out of a waveguide structure in integrated optics for detection. An on-chip PA will mitigate these losses before detection. On 
the other hand, the PA scheme can only be used in the final measurement of light and cannot be inserted into a quantum network to overcome losses. This is because the PA amplifies the fields to a macroscopic level that loses the quantum signature of the original input states. Furthermore, the scheme only works for measurement of continuous variables such as homodyne detection but fails in photon-counting technique.

\section{ACKNOWLEDGMENT}

This work was supported by U.S. National Science Foundation (Grant No. 1806425).

\section{APPENDIX}

\section{Derivation of the input-output relation of the Wigner} function for a parametric amplifier [Eq. (6)]

To find the input-output relation, we consider the twomode Wigner function of a system in the state described by density operator $\hat{\rho}$, which is defined through the characteristic function

$$
\begin{aligned}
& \chi\left(u_{1}, v_{1} ; u_{2}, v_{2}\right) \\
& \quad=\operatorname{Tr}\left(\hat{\rho} e^{j v_{1} \hat{X}_{1}-j u_{1} \hat{Y}_{1}+j v_{2} \hat{X}_{2}-j u_{2} \hat{Y}_{2}}\right) .
\end{aligned}
$$

The Wigner function is a Fourier transformation of the characteristic function

$$
\begin{aligned}
W\left(x_{1}, y_{1} ; x_{2}, y_{2}\right)= & \frac{1}{(2 \pi)^{2}} \int d x_{1} d y_{1} d x_{2} d y_{2} \chi\left(u_{1}, v_{1} ; u_{2}, v_{2}\right) \\
& \times e^{j u_{1} y_{1}-j v_{1} x_{1}+j u_{2} y_{2}-j v_{2} x_{2}} .
\end{aligned}
$$

Assume the input state is described by a Wigner function $W_{\text {in }}\left(X_{1}, Y_{1} ; X_{2}, Y_{2}\right)$ for a parametric amplifier. Since the inputoutput relations presented in Eqs. (4) and (5) are for the Heisenberg picture, the state described by a density operator $\hat{\rho}$ is the same for both the input and output. Using Eq. (4), we find

$$
\begin{aligned}
\chi_{\text {out }}\left(u_{1}, v_{1} ; u_{2}, v_{2}\right) & =\operatorname{Tr}\left(\hat{\rho} e^{j v_{1} \hat{X}_{1}^{(o)}-j u_{1} \hat{Y}_{1}^{(o)}+j v_{2} \hat{X}_{2}^{(o)}-j u_{2} \hat{Y}_{2}^{(o)}}\right) \\
& =\operatorname{Tr}\left(\hat{\rho} e^{j v_{1}^{\prime} \hat{X}_{1}^{(i)}-j u_{1}^{\prime} \hat{Y}_{1}^{(i)}+j v_{2}^{\prime} \hat{X}_{2}^{(i)}-j u_{2}^{\prime} \hat{Y}_{2}^{(i)}}\right) \\
& =\chi_{\text {in }}\left(v_{1}^{\prime}, u_{1}^{\prime} ; v_{2}^{\prime}, u_{2}^{\prime}\right)
\end{aligned}
$$

where $u_{1}^{\prime}=u_{1} G-u_{2} g, \quad v_{1}^{\prime}=v_{1} G+v_{2} g, \quad u_{2}^{\prime}=u_{2} G-u_{1} g$, and $v_{2}^{\prime}=v_{2} G+v_{1} g$. Taking the reverse Fourier transformation for $W$, we find

$$
\begin{aligned}
W_{\mathrm{out}}^{(\mathrm{PA})}\left(x_{1}, y_{1} ; x_{2}, y_{2}\right)= & W_{\mathrm{in}}\left(G x_{1}-g x_{2}, G y_{1}+g y_{2} ; G x_{2}\right. \\
& \left.-g x_{1}, G y_{2}+g y_{1}\right) .
\end{aligned}
$$

\section{Derivation of Eqs. (27) and (33)}

For the scheme with a beam splitter for the Bell measurement, we have, from Eq. (26),

$$
W_{\text {out }}(X, Y)=\int W_{\text {in }}(X-x, Y-y) G_{\bar{\sigma}_{1}}(x, y) d x d y .
$$

In terms of the Wigner function, the density matrix is

$$
\begin{aligned}
\hat{\rho}_{\text {out }}(\hat{X}, \hat{Y}) & =\frac{1}{\pi} \int W_{\text {out }}(X, Y) e^{j v(\hat{X}-X)+j u(\hat{Y}-Y)} d v d u d X d Y \\
& =\frac{1}{\pi} \int W_{\text {in }}(X-x, Y-y) G_{\bar{\sigma}_{1}}(x, y) e^{j v(\hat{X}-X)+j u(\hat{Y}-Y)} d v d u d X d Y d x d y .
\end{aligned}
$$

Now let us shift $X$ and $Y$ in $e^{j v(\hat{X}-X)+j u(\hat{Y}-Y)}$ to $X-x$ and $Y-y$ by using the operator $\hat{D}(\alpha) \equiv \exp \left(\alpha \hat{a}^{\dagger}-\alpha^{*} \hat{a}\right): \hat{D}(\alpha) \hat{a} \hat{D}^{\dagger}(\alpha)=$ $\hat{a}-\alpha$. With $\alpha=(x+j y) / 2$ we have

$$
\begin{aligned}
\hat{\rho}_{\text {out }}(\hat{X}, \hat{Y})= & \frac{1}{\pi} \int W_{\text {in }}(X-x, Y-y) G_{\bar{\sigma}_{1}}(x, y) \hat{D}\left(\frac{x+y j}{2}\right) e^{j v[\hat{X}-(X-x)]+j u[\hat{Y}-(Y-y)]} \\
& \times \hat{D}^{\dagger}\left(\frac{x+y j}{2}\right) d v d u d X d Y d x d y .
\end{aligned}
$$

Making a change of variables $X-x, Y-y \rightarrow X, Y$ in the integral with respect to $X, Y$, we have

$$
\begin{aligned}
\hat{\rho}_{\text {out }}(\hat{X}, \hat{Y})= & \frac{1}{\pi} \int W_{\text {in }}(X-x, Y-y) G_{\bar{\sigma}_{1}}(x, y) \\
& \times \hat{D}\left(\frac{x+y j}{2}\right) e^{j v[\hat{X}-(X-x)]+j u[\hat{Y}-(Y-y)]} \hat{D}^{\dagger}\left(\frac{x+y j}{2}\right) d v d u d(X-x) d(Y-y) d x d y \\
= & \frac{1}{\pi} \int G_{\bar{\sigma}_{1}}(x, y) \hat{D}\left(\frac{x+y j}{2}\right) W_{\text {in }}(X, Y) e^{j v(\hat{X}-X)+j u(\hat{Y}-Y)} d v d u d X d Y \hat{D}^{\dagger}\left(\frac{x+y j}{2}\right) d x d y \\
= & \int G_{\bar{\sigma}_{1}}(x, y) \hat{D}\left(\frac{x+y j}{2}\right) \hat{\rho}_{\text {in }}(\hat{X}, \hat{Y}) \hat{D}^{\dagger}\left(\frac{x+y j}{2}\right) d x d y,
\end{aligned}
$$

which is just Eq. (27). 
For the scheme with a parametric amplifier for the Bell measurement, we have, from Eq. (32),

$$
W_{\text {out }}(X, Y)=\int W_{\text {in }}\left(k X-x, \frac{Y}{k}-y\right) G_{\bar{\sigma}_{2 x}}(x) G_{\bar{\sigma}_{2 y}}(y) d x d y,
$$

where $k \equiv g / G$. Defining $W_{\text {out }}^{\prime} \equiv W_{\text {in }} \circ G_{\bar{\sigma}_{2 x}} G_{\bar{\sigma}_{2 x}}$, we have

$$
W_{\text {out }}(X, Y)=W_{\text {out }}^{\prime}\left(k X, \frac{Y}{k}\right)=\frac{1}{2 \pi} \int d u\left\langle k X+u\left|\hat{\rho}_{\text {out }}^{\prime}\right| k X-u\right\rangle e^{-j u(Y / k)} .
$$

Making a change of $U=u / k$ in Eq. (A10), we have

$$
\begin{aligned}
W_{\text {out }}(X, Y) & =\frac{k}{2 \pi} \int d U\left\langle k X+k U\left|\hat{\rho}_{\text {out }}^{\prime}\right| k X-k U\right\rangle e^{-j U Y} \\
& =\frac{1}{2 \pi} \int d U\left\langle X+U\left|\hat{S}(\epsilon) \hat{\rho}_{\text {out }}^{\prime} \hat{S}^{\dagger}(\epsilon)\right| X-U\right\rangle e^{-j U Y},
\end{aligned}
$$

where $\epsilon=-\ln (k)=\ln (G / g)$. From Eqs. (A8) and (A10) we have

$$
\hat{\rho}_{\text {out }}^{\prime}=\int \hat{D}\left(\frac{x+y j}{2}\right) \hat{\rho}_{\text {in }} \hat{D}^{\dagger}\left(\frac{x+y j}{2}\right) G_{\bar{\sigma}_{2 x}}(x) G_{\bar{\sigma}_{2 y}}(y) d x d y .
$$

Therefore, we obtain Eq. (33),

$$
\hat{\rho}_{\text {out }}=\int \hat{S}(\epsilon) \hat{D}\left(\frac{x+y j}{2}\right) \hat{\rho}_{\text {in }} \hat{D}^{\dagger}\left(\frac{x+y j}{2}\right) \hat{S}^{\dagger}(\epsilon) G_{\bar{\sigma}_{2 x}}(x) G_{\bar{\sigma}_{2 y}}(y) d x d y .
$$

\section{Fidelity for coherent states with finite $s$ and $G$}

The PA scheme requires extra resources and large $G$ to work. We also need to have a large $s$ for the EPR entangled source for both BS and PA schemes. What is the relationship between $G$ and $s$ ?

To answer this, we calculate the fidelity $F$ for the coherent state input with no approximation applied to $G$ and $s$. We start from Eq. (23), but make the required displacement operation to obtain the final output state as

$$
W_{\text {out }}(x, y)=C \int d x^{\prime} d y^{\prime} e^{-(1 / 2 \cosh 2 s)\left(x^{2}+y^{2}\right)} W_{\text {in }}\left(x^{\prime}, y^{\prime}\right) G_{\sigma_{2 x}}\left(x^{\prime \prime}-x^{\prime}\right) G_{\sigma_{2 y}}\left(y^{\prime \prime}-y^{\prime}\right),
$$

with $x^{\prime \prime} \equiv x \frac{g}{G} \tanh 2 s, y^{\prime \prime} \equiv y \frac{G}{g} \tanh 2 s, \sigma_{2 x}^{2}=\frac{1-\eta^{2}}{\eta^{2} G^{2}}+\frac{g^{2}}{G^{2} \cosh 2 s}$, and $\sigma_{2 y}^{2}=\frac{1-\eta^{2}}{\eta^{2} g^{2}}+\frac{G^{2}}{g^{2} \cosh 2 s}$ for the PA case. Here $C$ is a normalization constant. The BS case can be derived in a similar procedure leading to Eqs. (20) and (23) but without an $s \gg 1$ approximation and has the same form as Eq. (A14) but with $x^{\prime \prime} \equiv x \tanh 2 s, y^{\prime \prime} \equiv y \tanh 2 s$, and $\sigma_{2 x}^{2}$ and $\sigma_{2 y}^{2}$ both replaced by $\sigma_{1}=2 \frac{1-\eta^{2}}{\eta^{2}}+\frac{1}{\cosh 2 s}$. Thus, the PA and BS schemes are equivalent as long as $G \approx g$ or $G \gg 1$. This condition is independent of the value of $s$. Of course, the PA scheme has the advantage of reducing the effect of losses by a factor of $2 G^{2}$.

We next evaluate $F$ from Eq. (25) for a coherent state input. The results are

$$
\begin{aligned}
F_{B S} & =\frac{2\left(\tanh ^{2} 2 s+\frac{\sigma_{1}^{2}+1}{\cosh 2 s}\right)}{\tanh ^{2} 2 s+\sigma_{1}^{2}+1+\frac{\sigma_{1}^{2}+1}{\cosh 2 s}} \exp \left(\frac{-2\left(a^{2}+b^{2}\right)\left[(1-\tanh 2 s)^{2}+\frac{\sigma_{1}^{2}+2}{\cosh 2 s}\right]}{\tanh ^{2} 2 s+\sigma_{1}^{2}+1+\frac{\sigma_{1}^{2}+1}{\cosh 2 s}}\right) \exp \left(\frac{\frac{2\left(a^{2}+b^{2}\right)}{\cosh 2 s}}{\tanh ^{2} 2 s+\frac{\sigma_{1}^{2}+1}{\cosh 2 s}}\right) \\
& \approx \frac{1}{1+\bar{\sigma}_{1}^{2} / 2} \quad \text { for } s \gg 1,
\end{aligned}
$$

with $\bar{\sigma}_{1}=2 \frac{1-\eta^{2}}{\eta^{2}}$, and

$$
\begin{aligned}
F_{P A}= & \sqrt{\frac{\frac{g^{2} \tanh ^{2} 2 s}{G^{2}}+\frac{\sigma_{2 x}^{2}+1}{\cosh 2 s}}{\frac{g^{2} \tanh ^{2} 2 s}{G^{2}}+\left(\sigma_{2 x}^{2}+1\right)\left(1+\frac{1}{\cosh 2 s}\right)}} \sqrt{\frac{\frac{G^{2} \tanh ^{2} 2 s}{g^{2}}+\frac{\sigma_{2 y}^{2}+1}{\cosh 2 s}}{\frac{G^{2} \tanh ^{2} 2 s}{g^{2}}+\left(\sigma_{2 y}^{2}+1\right)\left(1+\frac{1}{\cosh 2 s}\right)}} \\
& \times \exp \left(\frac{-2 a^{2}\left[\left(1-\frac{g \tanh 2 s}{G}\right)^{2}+\frac{\sigma_{2 x}^{2}+2}{\cosh (2 s)}\right]}{\frac{g^{2} \tanh ^{2} 2 s}{G^{2}}+\left(\sigma_{2 x}^{2}+1\right)\left(1+\frac{1}{\cosh 2 s}\right)}\right) \exp \left(\frac{-2 b^{2}\left[\left(1-\frac{G \tanh 2 s}{g}\right)^{2}+\frac{\sigma_{2 y}^{2}+2}{\cosh (2 s)}\right]}{\frac{G^{2} \tanh ^{2} 2 s}{g^{2}}+\left(\sigma_{2 y}^{2}+1\right)\left(1+\frac{1}{\cosh 2 s}\right)}\right) \\
& \times \exp \left(\frac{\frac{2 a^{2}}{\cosh 2 s}}{\frac{g^{2} \tanh ^{2} 2 s}{G^{2}}+\frac{\sigma_{x}^{2}+1}{\cosh 2 s}}+\frac{\frac{2 b^{2}}{\cosh 2 s}}{\frac{G^{2} \tanh ^{2} 2 s}{g^{2}}+\frac{\sigma_{2 y}^{2}+1}{\cosh 2 s}}\right) .
\end{aligned}
$$


If $s \gg 1$ so that $\tanh 2 s \approx 1$ and $1 / \cosh 2 s \ll 1$ (independent of the value of $G$ ), the expression (A16) becomes Eq. (36). On the other hand, if $G \gg 1$ so that $G \approx g$ and $\sigma_{2 x}^{2} \approx \sigma_{2 y}^{2} \approx \sigma_{2}^{2} \equiv \frac{1-\eta^{2}}{\eta^{2} G^{2}}+\frac{1}{\cosh 2 s}$, we have

$$
\begin{aligned}
& F_{P A} \approx \frac{2\left(\tanh ^{2} 2 s+\frac{\sigma_{2}^{2}+1}{\cosh 2 s}\right)}{\tanh ^{2} 2 s+\sigma_{2}^{2}+1+\frac{\sigma_{2}^{2}+1}{\cosh 2 s}} \exp \left(\frac{-2\left(a^{2}+b^{2}\right)\left[(1-\tanh 2 s)^{2}+\frac{\sigma_{2}^{2}+2}{\cosh 2 s}\right]}{\tanh ^{2} 2 s+\sigma_{2}^{2}+1+\frac{\sigma_{2}^{2}+1}{\cosh 2 s}}\right) \exp \left(\frac{\frac{2\left(a^{2}+b^{2}\right)}{\cosh 2 s}}{\tanh ^{2} 2 s+\frac{\sigma_{2}^{2}+1}{\cosh 2 s}}\right) \\
& \approx \frac{1}{1+\bar{\sigma}_{2}^{2} / 2} \text { for } s \gg 1
\end{aligned}
$$

with $\bar{\sigma}_{2}=\frac{1-\eta^{2}}{\eta^{2} G^{2}}$. Note that the difference between Eqs. (A15) and (A17) is in $\sigma_{1}\left(\bar{\sigma}_{1}\right)$ and $\sigma_{2}\left(\bar{\sigma}_{2}\right)$ with $\sigma_{2}$ improved upon $\sigma_{1}$ by the reduction of the loss effect term by a factor of $2 G^{2}$.

Comparing the corresponding terms in Eqs. (A16) and (A17), we find that their differences are all smaller than $1 / G^{2}$, which is independent of $s$. Thus the limit of $G \gg 1$ is also independent of $s$.

[1] E. Knill, R. Laflamme, and G. J. Milburn, A scheme for efficient quantum computation with linear optics, Nature (London) 409, 46 (2001).

[2] P. Kok, W. J. Munro, K. Nemoto, T. C. Ralph, J. P. Dowling, and G. J. Milburn, Linear optical quantum computing with photonic qubits, Rev. Mod. Phys. 79, 135 (2007).

[3] S. L. Braunstein and A. Mann, Measurement of the Bell operator and quantum teleportation, Phys. Rev. A 51, R1727 (1995).

[4] S. L. Braunstein and H. J. Kimble, Teleportation of Continuous Quantum Variables, Phys. Rev. Lett. 80, 869 (1998).

[5] C. H. Bennett, G. Brassard, C. Crépeau, R. Jozsa, A. Peres, and W. K. Wootters, Teleporting an Unknown Quantum State via Dual Classical and Einstein-Podolsky-Rosen Channels, Phys. Rev. Lett. 70, 1895 (1993).

[6] L. Vaidman, Teleportation of quantum states, Phys. Rev. A 49, 1473 (1994).

[7] D. Bouwmeester, J.-W. Pan, K. Mattle, M. Eibl, H. Weinfurter, and A. Zeilinger, Experimental quantum teleportation, Nature (London) 390, 575 (1997).

[8] A. Furusawa, J. L. Sorensen, S. L. Braunstein, C. A. Fuchs, H. J. Kimble, and E. S. Polzik, Unconditional quantum teleportation, Science 282, 706 (1998).

[9] Z. Y. Ou, Enhancement of the phase-measurement sensitivity beyond the standard quantum limit by a nonlinear interferometer, Phys. Rev. A 85, 023815 (2012).

[10] J. Li, Y. Liu, N. Huo, L. Cui, C. Feng, Z. Y. Ou, and $\mathrm{X}$. Li, Pulsed entanglement measured by parametric amplifier assisted homodyne detection, Opt. Express 27, 30552 (2019).

[11] C. M. Caves, Quantum limits on noise in linear amplifiers, Phys. Rev. D 26, 1817 (1982).
[12] Z. Y. Ou, Quantum amplification with correlated quantum fields, Phys. Rev. A 48, R1761 (1993).

[13] J. Kong, F. Hudelist, Z. Y. Ou, and W. Zhang, Cancellation of Internal Quantum Noise of an Amplifier by Quantum Correlation, Phys. Rev. Lett. 111, 033608 (2013).

[14] U. Leohardt, Measuring the Quantum State of Light (Cambridge University Press, Cambridge, 1997).

[15] Z. Y. Ou, Quantum Optics for Experimentalists (World Scientific, Singapore, 2017).

[16] Z. Y. Ou, S. F. Pereira, and H. J. Kimble, Realization of the Einstein-Podolsky-Rosen paradox for continuous variables in nondegenerate parametric amplification, Appl. Phys. B 55, 265 (1992).

[17] S. L. Braunstein and H. J. Kimble, A posteriori teleportation, Nature (London) 394, 840 (1998).

[18] R. E. S. Polkinghorne and T. C. Ralph, Continuous Variable Entanglement Swapping, Phys. Rev. Lett. 83, 2095 (1999).

[19] P. van Loock and S. L. Braunstein, Unconditional teleportation of continuous-variable entanglement, Phys. Rev. A 61, 010302(R) (1999).

[20] O. Glöckl, S. Lorenz, C. Marquardt, J. Heersink, M. Brownnutt, C. Silberhorn, Q. Pan, P. van Loock, N. Korolkova, and G. Leuchs, Experiment towards continuous-variable entanglement swapping: Highly correlated four-partite quantum state, Phys. Rev. A 68, 012319 (2003).

[21] R. Jozsa, Fidelity for mixed quantum states, J. Mod. Opt. 41, 2315 (1994).

[22] B. Schumacher, Sending entanglement through noisy quantum channels, Phys. Rev. A 54, 2614 (1996).

[23] L.-M. Duan, G. Giedke, J. I. Cirac, and P. Zoller, Inseparability Criterion for Continuous Variable Systems, Phys. Rev. Lett. 84, 2722 (2000). 